\title{
CONTENTS
}

Special issue dedicated to the memory of prof. Iliya Blekhman

Alexander Fradkov and Dmitry Indejtsev

Preface. Special issue dedicated to the memory of Prof. Iliya Blekhman

Grigory Altshul, Alexander Gouskov, Grigory Panovko, and Alexander Shokhin

Dynamics features of a vibrating machine with elastic element having exponential characteristic of resilient

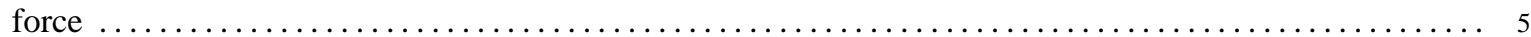

Boris Andrievsky and Vladimir I. Boikov

Bidirectional controlled multiple synchronization of unbalanced rotors and its experimental evaluation ....

Sergey P. Gorbikov

Conditions for topological equivalence of local qualitative singularities of dynamical systems with impact

interactions

Eugen Kremer

Vibratonal mechanics and stochastic quasi-resonances

Konstantin Lurie

Material optimization and dynamic materials

Alexey Smirnov and Boris Smolnikov

Collinear control of oscillation modes of spatial double pendulum with variable gain

O.P. Tomchina, D.V.Gorlatov, D.A.Tomchin, and A.E.Epishkin

Control of passage through resonance zone for 1-rotor vibration unit with time-varying load

Nikolay P. Yaroshevich, Tetjana S. Yaroshevych, and Olexander V. Shovkomud

Slow oscillations in drives of machine assemblies 


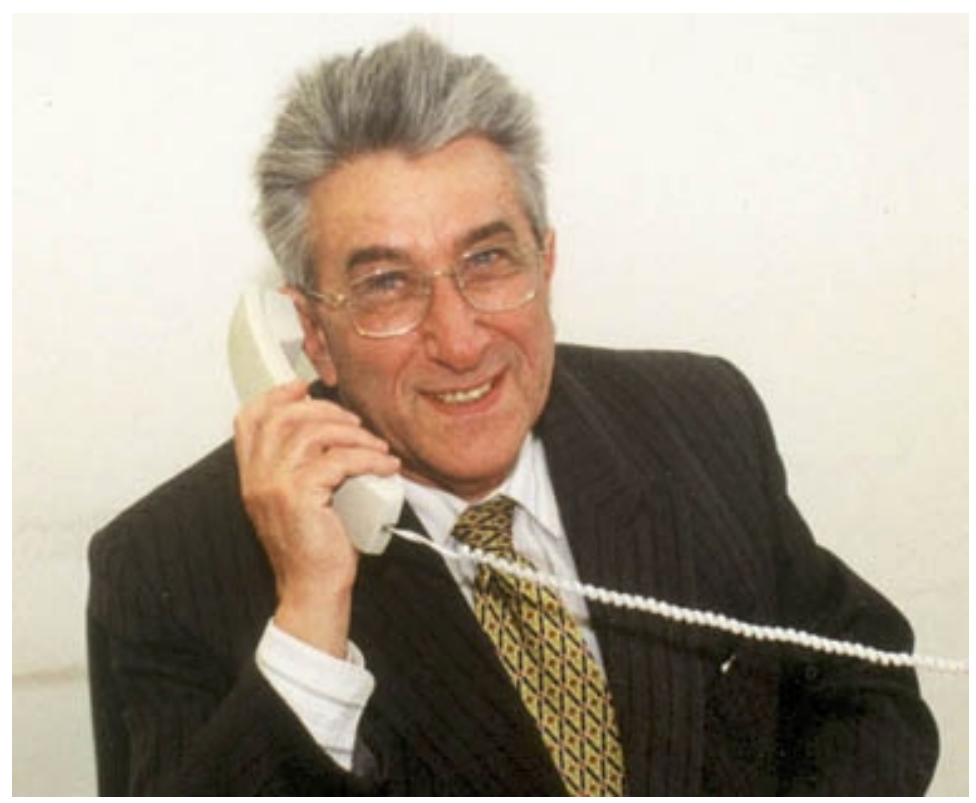

\section{Preface. Special issue dedicated to the memory of Prof. Iliya Blekhman}

Without passion there are no great scientists, no great artists, no great philosophers

Helvetii

What was said by the famous French philosopher surprisingly accurately characterizes the main character traits of Professor Iliya Blekhman. The passion for the knowledge of the deep laws of nature, close attention to everything new that surrounded him, the desire to listen to his opponent and try to take his position - all this allowed Iliya Izraelevich to say his weighty new word in the science of mechanics and oscillation theory. It was he who managed to realize and clearly formulate the need for a new theoretical approach to the oscillations of nonlinear systems, to develop a "new nonlinear oscillatory thinking". The separation of movements into fast and slow ones, "successfully", which allowed Reynolds to introduce tangential stresses characteristic of turbulent processes, found its place to obtain new elegant mathematical models describing the dynamics of media with complex rheology. Surprisingly, ingeniously simple models made it possible to understand fundamentally new physical phenomena characteristic both for the macro- and for microcosm.

Iliya Izraelevich Blekhman, a leading Russian expert in the areas of nonlinear oscillations, the dynamics of machines and vibration technology was born on 29 November 1928 in Kharkov (now in Ukraine) and completed five years of secondary school in Leningrad (now Saint Petersburg) before World War II. During the blockade of the city in 1942 he was evacuated to the Urals, to Sverdlovsk (now Ekaterinburg) and, after passing two intermediate examinations as an external candidate, graduated from school with a gold medal award. Between 1945 and 1947 he studied at the Mechanics Department of the Urals Polytechnic Institute and, at the same time, at the Mechanics and Mathematics Faculty of the Urals State University as an external student. In 1947 he entered the Physics and Mathematics Faculty of Leningrad Polytechnic Institute, and graduated with distinction as a research engineer in technical mechanics in 1951. His advisor was famous expert in mechanics and nonlinear control Anatolii Isakovich Lurie who later founded the Department of Mechanics and Control processes in Leningrad Polytechnic University. In 1955 he successfully defended his candidate's thesis, in 1962 - his doctoral dissertation, and in 1969 he was awarded the title of professor.

I. I. Blekhman's scientific and engineering activity began and continues at the Mekhanobr Institute, where he began working back in 1949 during his student internship. Here he organized a computational and theoretical service in the field of mechanics and applied mathematics. Since 1996, it has been a joint laboratory of the NPK "MekhanobrTechnika" and the Institute of Problems in Mechanical Engineering of the Russian Academy of Sciences (IPME RAS). 
Among his most significant results in these areas are:

- creation and development of two new sections of the theory of nonlinear oscillations and rheology - vibrational mechanics and vibrational rheology, which have received wide international recognition and development;

- creation (together with G. Yu. Janelidze) of the theory of vibrational displacement — the process of "directed" slow changes occurring under the influence of fast "non-directional" influences;

- detection (together with colleagues at the Mechanobr Institute) of the phenomenon of self-synchronization of rotating bodies (rotors) and creation of a theory of this phenomenon;

- establishment of an extreme sign of stability extending the Lagrange-Dirichlet theorem on the stability of equilibrium positions to synchronous rotations of weakly interacting bodies;

- description and study of a class of mechanical systems that, under the influence of vibration, acquire the properties of "potentiality on average": slow movements in such systems correspond to the movements of some averaged potential system, whereas the original system is essentially non-potential;

- generalization of the classical Laval auto-balancing principle to multi-rotor and nonlinear systems;

- detection (together with colleagues at the Mekhanobr Institute) and theoretical substantiation of the phenomenon of the occurrence of a gravity-gas-lift flow (fountain), which opens up effective ways to develop energy and raw materials resources of the World Ocean and prevent environmental disasters;

- experimental detection and theoretical explanation (together with staff and students) of the phenomenon of vibrational injection of gas into a liquid;

- promotion (together with K.A. Lurie) and development of the idea of creating fundamentally new materials the so-called dynamic materials and composites.

A significant part of these results has been reflected not only in scientific, but also in reference and educational literature and has found wide practical application.

The discovered, theoretically substantiated and described phenomena of synchronization of rotating bodies, the occurrence of gravitational gas lift flow and vibrational injection of gas into a liquid are registered as scientific discoveries.

Jointly with A. D. Myshkis and Ya. G. Panovko, I. I. Blekhman has written a monograph on the methodological features of mechanics and applied mathematics, which has became widely known and went through six editions. This unique book has an amazing mixed flavor of philosophy and humor providing readers with passion, not only with the knowledge. He was the author of a number of sections of the 2 nd and 4th volumes of the encyclopedic handbook "Vibrations in Technology" and the editor of the 2nd volume.

I. I. Blekhman owns a number of important engineering developments and patents for the creation of new vibrating machines for processing natural and man-made materials - crushers, mills, screens, flotation machines, concentration tables, conveyors, feeders, etc.

I. I. Blekhman and his followers received more than 500 certificates for inventions and patents, sold licenses to leading firms in the USA, Japan, Iran and other countries. He has published more than 400 research works, including 15 monographs, three of which have been republished in the USA, England and Germany.

I. I. Blekhman created a scientific school in the field of the theory of vibration processes and machines, recognized as the leading scientific school in Russia. He has trained 10 doctors and more than 50 candidates of sciences.

I. I. Blekhman played an important role in promoting activities in the area related to Physics (Mechanics) and Control Systems. He was one of the first researchers who recognized great potential of cooperation between experts in Physics and Control. He clearly understood specific features for the boundary scientific area between Physics and Control. His kind and unwavering support meant a lot to those who laid the foundations of a new scientific field. It is important that he actively participated in the work of the Advisory Board of Cybernetics And Physics Journal and was always happy to help the organizers.

This Special Issue is dedicated to the memory and achievements of Iliya Izraelevich Blekhman. It contains some papers related to some extent to the huge area of his research interests. More biographical and research materials related to life and works of Professor Blekhman as well as the list of his publications can be found of the website of IPME: http://ipme.ru 